\title{
Direct N-Acetyl Enamine Formation: \\ Lithium Bromide Mediated Addition of Methyllithium to Nitriles
}

Cécile G. Savarin,* Geneviève N. Boice, Jerry A. Murry, Edward

Corley, Lisa DiMichele and Dave Hughes

Department of Process Research, Merck Research Laboratories, Merck \& Co., P.O. Box 2000, Rahway, New Jersey 07065

Cecile_savarin@merck.com

General Methods. Reagents and solvents were obtained from commercial suppliers and were used without further purification or drying unless otherwise noted. Chromatography was done on silica gel (70$230 \mathrm{mesh}) .{ }^{1} \mathrm{H} \mathrm{NMR}$ and ${ }^{13} \mathrm{C}$ NMR spectra were recorded at 400 or 500 and $100 \mathrm{MHz}$, respectively as stated in the text.

General Procedure. Enamide Formation using MeLi.LiBr as a complex

To a flask under $\mathrm{N}_{2}$ is added nitrile and MTBE (12 volumes). The homogeneous solution is cooled to -30 ${ }^{\circ} \mathrm{C}\left(+/-5^{\circ} \mathrm{C}\right)$ and MeLi.LiBr $\left(1.5 \mathrm{M}\right.$ in Et2O) is added at a rate such that the temperature remains $<-10^{\circ} \mathrm{C}$. After a 30-60 minute age, the solution is further cooled to $-50^{\circ} \mathrm{C}\left(+/-5^{\circ} \mathrm{C}\right)$. Acetic anhydride in $\mathrm{MTBE}$ $(3.4 \mathrm{M})$ is added at a rate such that the temperature remains $<-35^{\circ} \mathrm{C}$. The reaction is then allowed to warm to room temperature and aged overnight. MTBE and $6.5 \mathrm{wt} \% \mathrm{~K}_{2} \mathrm{HPO}_{4}(\mathrm{aq})$ are added. The mixture is stirred, the layers allowed to settle, and the aqueous layer is cut. The organic layer is washed with $6.5 \mathrm{wt} \%$ $\mathrm{K}_{2} \mathrm{HPO}_{4}(\mathrm{aq})$. The solution is solvent switched to EtOH and $5 \mathrm{~N} \mathrm{NaOH}$ is added until the apparent $\mathrm{pH}=12$ and the bis -acetylated compound is consumed. The reaction mixture is either concentrated to dryness and then submitted to column chromatography on silica gel or the enamide is isolated directly by crystallization (EtOAc or MTBE with heptane or hexanes crystallization - alternatively, EtOH: $\mathrm{H}_{2} \mathrm{O}$ crystallization can be done and is highly desirable for better subsequent asymmetric hydrogenation at low catalyst loading). In the case of EtOH: $\mathrm{H}_{2} \mathrm{O}$ crystallization, $\mathrm{H}_{2} \mathrm{O}$ is slowly added to a solution of the enamide in EtOH at $\mathrm{rt}$ (seeding the solution saturation point),<smiles>C=C(NC(C)=O)c1cc(Cl)ccc1C1CCN(C(=O)OC(C)(C)C)CC1</smiles>

4-[2-(1-Acetylaminovinyl)-4-chlorophenyl]-piperidine-1-carboxylic acid tert-butyl ester, $1 \mathbf{a}$ 
Crystallization from EtOH: $\mathrm{H}_{2} \mathrm{O}, 2: 1$ afforded the desired product as white crystals (1.5 g, 80\%). mp 213$215^{\circ} \mathrm{C} ;{ }^{1} \mathrm{H}$ NMR $\left(\mathrm{CDCl}_{3}, 400 \mathrm{MHz}\right) \delta 7.28(\mathrm{dd}, J=8.4,2.2 \mathrm{~Hz}, 1 \mathrm{H}), 7.20(\mathrm{~d}, J=2.2 \mathrm{~Hz}, 1 \mathrm{H}), 7.17(\mathrm{~d}, J=$ $8.4 \mathrm{~Hz}, 1 \mathrm{H}), 7.07$ (br s, 1H), 5.97 (br s, 1H), 4.64 (br s, 1H), 4.16 (app. d, $J=12.8 \mathrm{~Hz}, 2 \mathrm{H}), 2.94$ (tt, $J=$ $11.6 \mathrm{~Hz}, 3.2 \mathrm{~Hz}, 1 \mathrm{H}), 2.78-2.62(\mathrm{~m}, 2 \mathrm{H}), 2.00(\mathrm{~s}, 3 \mathrm{H}), 1.67-1.51(\mathrm{~m}, 4 \mathrm{H}), 1.44(\mathrm{~s}, 9 \mathrm{H}) ;{ }^{13} \mathrm{C} \mathrm{NMR}\left(\mathrm{CDCl}_{3}\right.$, $100 \mathrm{MHz}) \delta 168.6,154.8,142.4,139.5,138.8,131.7,129.4,129.0,128.1,103.6,79.6,44.3,38.2,33.5$, 28.5, 24.4; Anal. Calcd for $\mathrm{C}_{20} \mathrm{H}_{27} \mathrm{ClN}_{2} \mathrm{O}_{3}$ : C, 63.40; H, 7.18; N, 7.39. Found: C, 63.59; H, 7.36; N, 7.61.

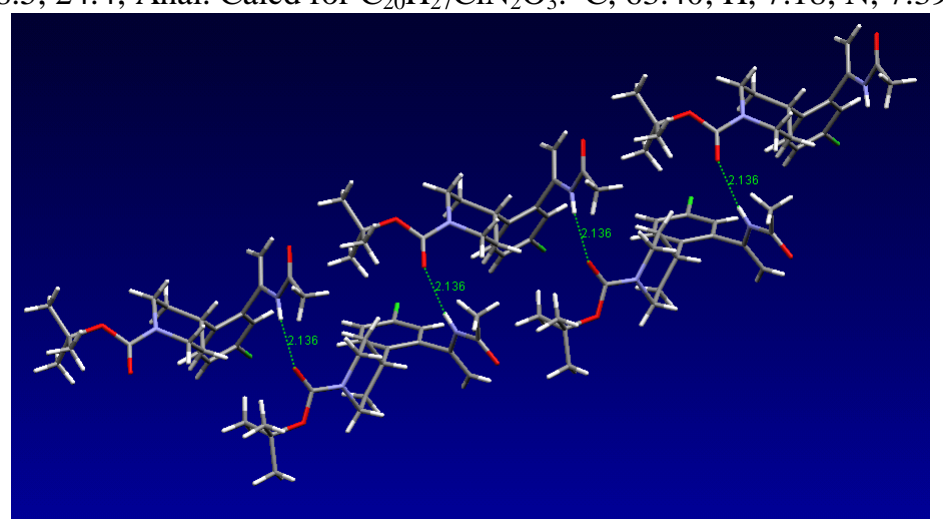

A single crystal was selected for single crystal x-ray data collection on a Bruker Smart Apex system. The crystal was colorless polyhedron with dimensions of $0.28 \mathrm{~mm} \times 0.26 \mathrm{~mm} \times 0.14 \mathrm{~mm}$. The unit cell was collected on 10 second scan rate and indexing gave the cell setting to be monoclinic. The structure was solved in the monoclinic P21/c space group after a full quadrant data collection using 10 second scan rate. See Table 1 for tabulated information pertaining to the final specifications of the solved structure.

\section{Table 1. Crystal data and structure refinement for jrc0931m.}

Identification code

Empirical formula

Formula weight

Temperature

Wavelength

Crystal system, space group

Unit cell dimensions

Volume

Z, Calculated density

Absorption coefficient

$\mathrm{F}(000)$

Crystal size

Theta range for data collection

Limiting indices

Reflections collected / unique

Completeness to theta $=23.25$

Absorption correction

Refinement method

Data / restraints / parameters

Goodness-of-fit on $\mathrm{F}^{\wedge} 2$

Final R indices [I $>2 \operatorname{sigma}(\mathrm{I})]$

$\mathrm{R}$ indices (all data)

Largest diff. peak and hole

\section{jrc0931m \\ $\mathrm{C} 20 \mathrm{H} 27 \mathrm{Cl} \mathrm{N} 2 \mathrm{O} 3$ \\ 378.89 \\ 298(2) K \\ $0.71073 \mathrm{~A}$}

Monoclinic,

$\mathrm{P} 2(1) / \mathrm{c}$

$$
\mathrm{a}=10.658(5) \text { A } \text { alpha }=90 \mathrm{deg} \text {. }
$$

$\mathrm{b}=15.006(7) \mathrm{A} \quad$ beta $=109.975(7) \mathrm{deg}$.

$\mathrm{c}=13.850(6) \mathrm{A}$ gamma $=90 \mathrm{deg}$.

2081.9(16) $\mathrm{A}^{\wedge} 3$

4 ,

$1.209 \mathrm{Mg} / \mathrm{m}^{\wedge} 3$

$0.204 \mathrm{~mm}^{\wedge}-1$

808

$0.28 \times 0.26 \times 0.14 \mathrm{~mm}$

2.03 to $23.25 \mathrm{deg}$.

$-11<=\mathrm{h}<=11$,

$-16<=\mathrm{k}<=16$,

$-15<=1<=15$

$16863 / 2991[\mathrm{R}(\mathrm{int})=0.0671]$

$100.0 \%$

None

Full-matrix least-squares on $\mathrm{F}^{\wedge} 2$

2991 / 0 / 239

1.074

$$
\begin{gathered}
\mathrm{R} 1=0.0426, \\
\mathrm{R} 1=0.0534,
\end{gathered}
$$

0.285 and -0.330 e. $\mathrm{A}^{\wedge}-3$ 
<smiles>C=C(NC(C)=O)c1cc(Cl)ccc1C</smiles>

$N$-(1[-3-chloro-5-tolyl]vinyl)-acetamide, $1 \mathrm{~b}$

Crystallization from EtOH: $\mathrm{H}_{2} \mathrm{O}$ afforded the desired product as white crystals (1.6 g, 90\%). mp 109-110 ${ }^{\circ} \mathrm{C} ;{ }^{1} \mathrm{H}$ NMR $\left(\mathrm{CDCl}_{3}, 400 \mathrm{MHz}\right) \delta$ 7.22-7.18 (m, 2H), $7.12(\mathrm{~d}, J=8.0 \mathrm{~Hz}, 1 \mathrm{H}), 6.93(\mathrm{br} \mathrm{s}, 1 \mathrm{H}), 5.95(\mathrm{~s}, 1 \mathrm{H})$, $4.67(\mathrm{~s}, 1 \mathrm{H}), 2.29(\mathrm{~s}, 3 \mathrm{H}), 1.99(\mathrm{~s}, 3 \mathrm{H}) ;{ }^{13} \mathrm{C} \mathrm{NMR}\left(\mathrm{CDCl}_{3}, 100 \mathrm{MHz}\right) \delta 168.8,140.0,139.3,134.3,131.7$, $131.4,129.0,128.4,103.2,24.3,19.0$.

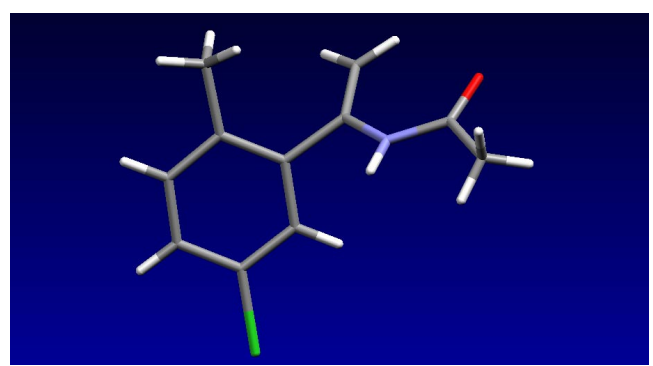

A single crystal was selected for single crystal x-ray data collection on a Bruker Smart Apex system. The crystal was pale yellow polyhedron with dimensions of $0.45 \mathrm{~mm} \times 0.25 \mathrm{~mm} \times 0.22 \mathrm{~mm}$. The unit cell was collected on 5 second scan rate and indexing gave the cell setting to be triclinic. The structure was solved in the triclinic P1 space group after a quadrant data collection using 5 second scan rate. See Table 1 for tabulated information pertaining to the final specifications of the solved structure.

Table 1

Identification code

$$
\begin{aligned}
& \text { jrc } 1001 \mathrm{~m} \\
& \mathrm{C}_{11} \mathrm{H}_{12} \mathrm{Cl} \mathrm{N} \mathrm{O} \\
& 209.67 \\
& 298(2) \mathrm{K} \\
& 0.71073 \mathrm{~A}
\end{aligned}
$$

Empirical formula

Formula weight

Temperature

Wavelength

Crystal system, space group

Unit cell dimensions

Triclinic,

$\mathrm{a}=9.577(3) \mathrm{A}$

$\mathrm{b}=11.401(3) \mathrm{A}$

$\mathrm{c}=11.847(3) \mathrm{A}$

1135.1(5) $\mathrm{A}^{3}$

$0.305 \mathrm{~mm}^{-1}$

$$
\text { 4, } 1.227 \mathrm{Mg} / \mathrm{m}^{3}
$$

440

$0.45 \times 0.25 \times 0.22 \mathrm{~mm}$

1.82 to $23.25 \mathrm{deg}$.

$$
\begin{aligned}
& -10<=\mathrm{h}<=10, \\
& -12<=\mathrm{k}<=12, \\
& -13<=\mathrm{l}<=13
\end{aligned}
$$

$9424 / 3269[\mathrm{R}(\mathrm{int})=0.2564]$

$100.0 \%$

None

Full-matrix least-squares on $\mathrm{F}^{2}$

3269 / 0 / 257

1.065

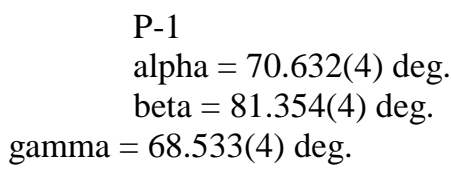

Completeness to theta $=23.25$

Absorption correction

Refinement method

Data / restraints / parameters

Goodness-of-fit on $\mathrm{F}^{2}$ 
Final $R$ indices $[\mathrm{I}>2 \operatorname{sigma}(\mathrm{I})]$

$\mathrm{R}$ indices (all data)

Largest diff. peak and hole<smiles>C=C(NC(C)=O)c1cccc(Cl)c1</smiles>

$$
\begin{array}{rr}
\mathrm{R} 1=0.0682, & \mathrm{wR} 2=0.1602 \\
\mathrm{R} 1=0.0906, & \mathrm{wR} 2=0.1760
\end{array}
$$$$
0.276 \text { and }-0.523 \text { e. } \mathrm{A}^{-3}
$$

$N$-[1-(3-chlorophenyl)-vinyl]-acetamide, $1 \mathrm{c}^{1}$

The enamide was purified via column chromatography on silica gel (2:1, Hexanes:EtOAc) to afford the desired product as a yellow oil $(19.88 \mathrm{~g}, 70 \%$ yield $) .{ }^{1} \mathrm{H} \mathrm{NMR}\left(\mathrm{CDCl}_{3}, 400 \mathrm{MHz}\right) \delta 7.40(\mathrm{~s}, 1 \mathrm{H}), 7.34-7.28$ (m, 2H), 6.99 (br s, 1H), 5.81 (br s, 1H), 5.11 (br s, 1H), 2.12 (s, 3H).<smiles>C=C(NC(C)=O)c1cccc(C)c1</smiles>

$N$-(1-m-tolylvinyl)-acetamide, $1 \mathrm{~d}^{2}$

The enamide was purified via column chromatography on silica gel (3:1, Hexanes:EtOAc) to afford the desired product as white solid $(5.43 \mathrm{~g}, 73 \%$ yield $)$. mp $103-105{ }^{\circ} \mathrm{C} ;{ }^{1} \mathrm{H} \mathrm{NMR}\left(\mathrm{CDCl}_{3}, 400 \mathrm{MHz}\right) \delta 7.28-$ $7.07(\mathrm{~m}, 5 \mathrm{H}), 5.82(\mathrm{~s}, 1 \mathrm{H}), 5.06(\mathrm{~s}, 1 \mathrm{H}), 2.37(\mathrm{~s}, 3 \mathrm{H}), 2.09(\mathrm{~s}, 3 \mathrm{H}) ;{ }^{13} \mathrm{C} \mathrm{NMR}\left(\mathrm{CDCl}_{3}, 100 \mathrm{MHz}\right) \delta 169.1$, 140.6, 138.3, 129.3, 128.5, 126.7, 123.1, 102.1, 24.4, 21.3; Anal. Calcd for $\mathrm{C}_{11} \mathrm{H}_{13} \mathrm{NO}$ : C, 75.40; H, 7.48; N, 7.99. Found: C, 75.43; H, 7.55; N, 7.93.<smiles>C=C(NC(C)=O)c1c(F)cccc1F</smiles>

\section{$N$-[1-(2,6-difluorophenyl)-vinyl]-acetamide, $1 \mathrm{e}^{3}$}

The enamide was purified via column chromatography on silica gel (3:1, Hexanes:EtOAc) to afford the desired product as white solid $(2.41 \mathrm{~g}, 85 \%$ yield $)$. mp $130-132{ }^{\circ} \mathrm{C} ;{ }^{1} \mathrm{H} \mathrm{NMR}\left(\mathrm{CDCl}_{3}, 400 \mathrm{MHz}\right) \delta 7.32-$ $7.24(\mathrm{~m}, 1 \mathrm{H}), 7.02-6.88(\mathrm{~m}, 3 \mathrm{H}), 6.06(\mathrm{~s}, 1 \mathrm{H}), 4.94(\mathrm{~s}, 1 \mathrm{H}), 2.04(\mathrm{~s}, 3 \mathrm{H})$.<smiles>C=C(NC(C)=O)c1cc(Cl)ccc1-c1ccncc1</smiles>

\section{N-[1-(5-Chloro-2-pyridin-4-yl-phenyl)-vinyl]-acetamide, if}

The enamide was purified via column chromatography on silica gel (1:4, Hexanes:EtOAc to 1:1:0.1 Hexanes:EtOAC:MeOH) to afford the desired product as white solid (0.25 g, $76 \%$ yield). mp $170-172{ }^{\circ} \mathrm{C}$;

${ }^{1} \mathrm{H}$ NMR $\left(\mathrm{CDCl}_{3}, 400 \mathrm{MHz}\right) \delta 8.55(\mathrm{dd}, J=4.4,1.6 \mathrm{~Hz}, 2 \mathrm{H}), 7.46(\mathrm{~d}, J=2.4 \mathrm{~Hz}, 1 \mathrm{H}), 7.41(\mathrm{dd}, J=8.0,2.4$

${ }^{1}$ Gridnev, H. D.; Yasutake, M.; Higashi, N.; Imamoto, T. J. Am. Chem. Soc. 2001, 123, 22, 5268-5276.

${ }^{2}$ Zhu, G.; Zhang, X. J. Org. Chem. 1998, 63, 25, 9590-9593.

${ }^{3}$ Burk, M. J.; Wang, Y. M.; Lee, J. R. J. Am. Chem. Soc. 1996, 118, 21, 5142-5143. 
$\mathrm{Hz}, 1 \mathrm{H}), 7.33(\mathrm{dd}, J=4.4,1.6 \mathrm{~Hz}, 2 \mathrm{H}), 7.24(\mathrm{~d}, J=8.0 \mathrm{~Hz}, 1 \mathrm{H}), 5.59(\mathrm{~s}, 1 \mathrm{H}), 4.84(\mathrm{~s}, 1 \mathrm{H}), 1.55(\mathrm{~s}, 3 \mathrm{H})$;

${ }^{13} \mathrm{C} \mathrm{NMR}\left(\mathrm{CDCl}_{3}, 100 \mathrm{MHz}\right) \delta 168.2,149.7,147.7,139.3,138.6,135.2,134.8,131.2,130.2,129.1,123.2$, 106.4, 23.5; HRMS 272.0716 for $\mathrm{C}_{15} \mathrm{H}_{13} \mathrm{ClN}_{2} \mathrm{O}$ (real - [M+H] 273.0800, 4 ppm error).<smiles>C=CNC(=C)NC(=C)c1cccs1</smiles>

$N$-(1-Thiophene-2-yl-vinyl)-acetamide, $\mathbf{1 f}^{4}$

The enamide was purified via column chromatography to afford the desired product as colorless oil $(0.3 \mathrm{~g}$, $60 \%$ yield); ${ }^{1} \mathrm{H}$ NMR $\left(\mathrm{CDCl}_{3}, 400 \mathrm{MHz}\right) \delta 7.56(\mathrm{br} \mathrm{s}, 1 \mathrm{H}), 7.18(\mathrm{~d}, J=4.0 \mathrm{~Hz}, 1 \mathrm{H}), 7.08(\mathrm{~d}, J=4.0 \mathrm{~Hz}$, $1 \mathrm{H}), 6.94(\mathrm{t}, J=4.0 \mathrm{~Hz}, 1 \mathrm{H}), 5.63(\mathrm{br} \mathrm{s}, 1 \mathrm{H}), 5.20(\mathrm{br} \mathrm{s}, 1 \mathrm{H}), 2.04(\mathrm{~s}, 3 \mathrm{H})$.<smiles>C=C(NC(C)=O)C12CC3CC(CC(C3)C1)C2</smiles>

$N$-(1-Adamantan-1-yl-vinyl)-acetamide, $1 \mathrm{~h}^{5}$

The enamide was purified via crystallization from EtOH: $\mathrm{H}_{2} \mathrm{O}, 2: 1$ to afford the desired product as a white solid (2.21 g, $79 \%$ yield). mp 155-156 ${ }^{\circ} \mathrm{C} ;{ }^{1} \mathrm{H} \mathrm{NMR}\left(\mathrm{CDCl}_{3}, 400 \mathrm{MHz}\right) \delta 6.45(\mathrm{br} \mathrm{s}, 1 \mathrm{H}), 5.54(\mathrm{br} \mathrm{s}, 1 \mathrm{H})$, 4.75 (br s, 1H), 2.09-2.05 (m, 6H), 1.77-1.65 (m, 12H).<smiles>C=C(NC(C)=O)c1ccc(OC)cc1</smiles>

$N$-[1-(4-Methoxyphenyl-vinyl]-acetamide, $1 \mathrm{i}^{6}$

The enamide was purified via column chromatography on silica gel to afford the desired product as a white solid (0.2 g, $62 \%$ yield). mp 105-106 ${ }^{\circ} \mathrm{C} ;{ }^{1} \mathrm{H} \mathrm{NMR}\left(\mathrm{CDCl}_{3}, 400 \mathrm{MHz}\right) \delta 7.35(\mathrm{~d}, J=8.2 \mathrm{~Hz}, 2 \mathrm{H}), 6.88(\mathrm{~d}$, $J=8.2 \mathrm{~Hz}, 2 \mathrm{H}), 6.81$ (br s, 1H), 5.77 (br s, 1H), 5.02 (br s, 1H), 3.82 (s, 3H), 2.13 (s, 3H).

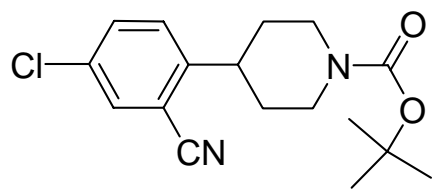

\section{tert-Butyl 4-(4-chloro-2-cyanophenyl)piperidine-1-carboxylate, 2a}

Prepared as previously described in the literature. ${ }^{7} \mathrm{mp} 97-99^{\circ} \mathrm{C} ;{ }^{1} \mathrm{H}$ NMR $\left(\mathrm{CDCl}_{3}, 400 \mathrm{MHz}\right) \delta 7.61(\mathrm{~d}$, $J=2.2 \mathrm{~Hz}, 1 \mathrm{H}), 7.55(\mathrm{dd}, J=8.6,2.2 \mathrm{~Hz}, 1 \mathrm{H}), 7.29(\mathrm{~d}, J=8.6 \mathrm{~Hz}, 1 \mathrm{H}), 4.28(\mathrm{br} \mathrm{s}, 2 \mathrm{H}), 3.11(\mathrm{tt}, J=12.2$, $3.5 \mathrm{~Hz}, 1 \mathrm{H}), 2.87(\operatorname{app~t}, J=12.0 \mathrm{~Hz}, 2 \mathrm{H}), 1.85(\operatorname{app~d}, J=13.1 \mathrm{~Hz}, 2 \mathrm{H}), 1.66-1.54(\mathrm{~m}, 2 \mathrm{H}), 1.49(\mathrm{~s}, 9 \mathrm{H})$; ${ }^{13} \mathrm{C}$ NMR $\left(\mathrm{CDCl}_{3}, 100 \mathrm{MHz}\right) \delta 154.5,147.5,133.3,132.5,132.4,127.8,116.5,113.3,79.6,43.9,40.4$,

\footnotetext{
${ }^{4}$ Minnaard, A. J.; Vries, A. H. de; Vries, J. G. de; Feringa, B. L. Adv. Synth. Catal. 2002, 344, 9, $1003-$ 1007.

${ }^{5}$ Burk, M. J.; Casy, G.; Johnson, N. B. J. Org. Chem. 1998, 63, 6084-6085.

${ }^{6}$ Zhu, G.; Zhang, X. J. Org. Chem. 1998, 63, 25, 9590-9593.

${ }^{7}$ Corley, E.; Conrad, K.; Murry, J.; Savarin, C.; Holko, J.; Boice, G. J. Org. Chem. 2004, 69, 5120-5123.
} 
32.2, 28.3; IR (thin film) 3072, 2975, 2929, 2858, 2235, $1681 \mathrm{~cm}^{-1}$; Anal. Calcd for $\mathrm{C}_{17} \mathrm{H}_{21} \mathrm{ClN}_{2} \mathrm{O}_{2}$ : $\mathrm{C}_{\text {, }}$ 63.64; H, 6.60; N, 8.73. Found: C, 63.85; H, 6.49; N, 8.60.<smiles>N#Cc1cc(Cl)ccc1-c1ccncc1</smiles>

\section{5-Chloro-2-pyridin-4-yl-benzonitrile, $2 f$}

A flask under $\mathrm{N}_{2}$ was charged with 2-bromo-5-chlorobenzonitrile 8 (2.1 g, $\left.9.6 \mathrm{mmol}\right)$, pyridine-4boronic acid $(2.0 \mathrm{~g}, 16.3 \mathrm{mmol}), \mathrm{K}_{3} \mathrm{PO}_{4}(4.7 \mathrm{~g}, 22.1 \mathrm{mmol}), \mathrm{Pd}\left(\mathrm{PPh}_{3}\right)_{4}(0.55 \mathrm{~g}, 0.5 \mathrm{mmol})$, Dioxane (90 $\mathrm{mL})$, and $\mathrm{H}_{2} \mathrm{O}(18 \mathrm{~mL})$. The reaction mixture was heated to $85^{\circ} \mathrm{C}$. A second charge of pyridine-4-boronic acid $(0.1 \mathrm{~g}, 0.8 \mathrm{mmol})$ was added after $5 \mathrm{~h}$. After $6 \mathrm{~h}$, the reaction mixture was cooled to rt. Saturated $\mathrm{NaHCO}_{3}(\mathrm{aq})(80 \mathrm{~mL})$ and EtOAc $(90 \mathrm{~mL})$ were added and the resulting layers were separated. The aqueous layer was extracted with EtOAc $(40 \mathrm{~mL})$ and the combined organic layers were dried over $\mathrm{Na}_{2} \mathrm{SO}_{4}$. The organic layer was concentrated to a golden oil. Purification by silica gel chromatography (2:1 EtOAc/Hexanes to $100 \%$ EtOAc) afforded the product as an off-white solid (1.69 g, 82\%). mp 143-145 ${ }^{\circ} \mathrm{C} ;{ }^{1} \mathrm{H} \mathrm{NMR}\left(\mathrm{CDCl}_{3}, 400 \mathrm{MHz}\right) \delta 8.75(\mathrm{dd}, J=4.5,1.7 \mathrm{~Hz}, 2 \mathrm{H}), 7.79(\mathrm{~d}, J=2.1 \mathrm{~Hz}, 1 \mathrm{H}), 7.68(\mathrm{dd}, J=$ 8.4, 2.1 Hz, 1H), 7.49-7.46 (m, 3H); ${ }^{13} \mathrm{C}$ NMR $\left(\mathrm{CDCl}_{3}, 100 \mathrm{MHz}\right) \delta 150.2,144.4,140.7,135.3,133.50$, 133.48, 131.0, 123.0, 116.5, 112.5; IR (thin film) 3350, 3065, 2228, $1598 \mathrm{~cm}^{-1}$; Anal. Calcd for $\mathrm{C}_{12} \mathrm{H}_{7} \mathrm{ClN}_{2}$ : C, 67.15; H, 3.29; N, 13.05. Found: C, 67.04; H, 2.96; N, 12.86.<smiles>CC(=O)/N=C(\C)c1cc(Cl)ccc1C1CCN(C(=O)OC(C)(C)C)CC1</smiles>

\section{4-(2-\{1-[-Acetylimino]-ethyl\}-4-chlorophenyl)-piperidine-1-carboxylic acid tert-butyl ester,} $4 \mathbf{a}$

${ }^{1} \mathrm{H}$ NMR $\left(\mathrm{CD}_{3} \mathrm{CN}, 500.13 \mathrm{MHz}\right) \delta 7.37(\mathrm{dd}, \mathrm{J}=8.3,2.4,1 \mathrm{H}), 7.33(\mathrm{~d}, \mathrm{~J}=8.3,1 \mathrm{H}), 7.31(\mathrm{~d}, \mathrm{~J}=2.4,1 \mathrm{H}), 4.15$ (br m, 2H), 2.94 (m, 1H), 2.73 (brm, 2H), 2.11 (s, 3H), 1.93 (s, 3H), 1.74 (br d, J=13.1, 2H), 1.55 (m, 2H), $1.44(\mathrm{~s}, 9 \mathrm{H}) ;{ }^{13} \mathrm{C}$ NMR $\left(\mathrm{CD}_{3} \mathrm{CN}, 125.77 \mathrm{MHz}\right) \delta 185.9,166.6,155.5,142.4,141.3,132.3,130.3,129.9$, 127.3, 79.9, 45.2 (broad, 2C), 39.5, 33.9 (2C), 28.7 (3C), 25.8, 25.4; ${ }^{15} \mathrm{~N} \mathrm{NMR}\left(\mathrm{CD}_{3} \mathrm{CN}, 50.69 \mathrm{MHz}\right) \delta$ 352 (footnote: BOC- $N$ too broad to observe)<smiles>C=C(N=[N+](C)[O-])c1cc(Cl)ccc1C1CCN(C(=O)OC(C)(C)C)CC1</smiles>

4-[4-Chloro-2-(1-diacetylaminovinyl)phenyl]-piperidine-1-carboxylic acid tert-butyl ester, $5 \mathbf{a}$

${ }^{1} \mathrm{H}$ NMR $\left(\mathrm{CDCl}_{3}, 400 \mathrm{MHz}\right) \delta 7.14(\mathrm{~s}, 2 \mathrm{H}), 6.97(\mathrm{~s}, 1 \mathrm{H}), 5.37(\mathrm{~s}, 1 \mathrm{H}), 5.24(\mathrm{~s}, 1 \mathrm{H}), 4.11(\mathrm{br}, 2 \mathrm{H}), 3.24(\mathrm{tt}$, $J=11.8,3.4 \mathrm{~Hz}, 1 \mathrm{H}), 2.63(\mathrm{t}, J=12.8 \mathrm{~Hz}, 2 \mathrm{H}), 2.30(\mathrm{~s}, 6 \mathrm{H}), 1.66-1.58(\mathrm{~m}, 2 \mathrm{H}), 1.57-1.44(\mathrm{~m}, 2 \mathrm{H}), 1.34(\mathrm{~s}$, $9 \mathrm{H}) ;{ }^{13} \mathrm{C} \mathrm{NMR}\left(\mathrm{CDCl}_{3}, 100 \mathrm{MHz}\right) \delta 172.7,154.7,143.2,141.5,137.4,131.5,129.2,128.8,126.0,119.7$, 79.4, 37.4, 33.7, 28.4, 26.2; ${ }^{15} \mathrm{~N}$ NMR $\left(\mathrm{CD}_{3} \mathrm{CN}, 50.69 \mathrm{MHz}\right) \delta \sim 193$. (footnote: BOC- $N$ too broad to observe) 


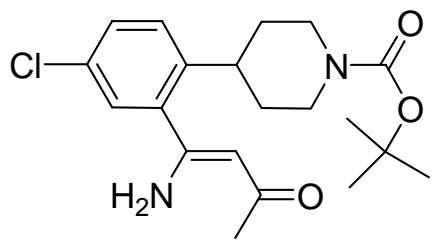

\section{4-[2-(-1-Amino-3-oxo-but-1-Z-enyl)-4-chlorophenyl]-piperidine-1-carboxylic acid tert-butyl ester, 6a \\ ${ }^{1} \mathrm{H}$ NMR $\left(\mathrm{CD}_{3} \mathrm{CN}, 500.13 \mathrm{MHz}\right) \delta 9.64(\mathrm{br} \mathrm{s}, 1 \mathrm{H}), 7.38(\mathrm{dd}, \mathrm{J}=8.3,2.4,1 \mathrm{H}), 7.34(\mathrm{~d}, \mathrm{~J}=8.3,1 \mathrm{H}), 7.25(\mathrm{~d}$, $\mathrm{J}=2.4,1 \mathrm{H}), 5.85($ br s. $1 \mathrm{H}), 5.04(\mathrm{~s}, 1 \mathrm{H}), 4.13(\mathrm{br} \mathrm{d}, \mathrm{J}=11.9,2 \mathrm{H}), 2.97(\mathrm{~m} .1 \mathrm{H}), 2.72(\mathrm{br} \mathrm{m}, 2 \mathrm{H}), 2.02$ (s, $3 \mathrm{H}), 1.69(\mathrm{~m}, 2 \mathrm{H}), 1.56(\mathrm{~m}, 2 \mathrm{H}), 1.44(\mathrm{~s}, 9 \mathrm{H}) ;{ }^{13} \mathrm{C} \mathrm{NMR}\left(\mathrm{CD}_{3} \mathrm{CN}, 125.77 \mathrm{MHz}\right) \delta 197.7,161.2,155.6$, 143.2, 139.8, 132.0, 130.2, 129.6, 128.9, 97.6, 79.9, 45.2 (2C), 39.3, 34.2 (2C), 29.9, 28.7 (3C), ${ }^{15} \mathrm{~N}$ NMR $\left(\mathrm{CD}_{3} \mathrm{CN}, 50.69 \mathrm{MHz}\right) \delta \sim 96$ (footnote: $B O C$ - $N$ too broad to observe)}<smiles>CC(=O)c1cc(Cl)ccc1C1CCN(C(=O)OC(C)(C)C)CC1</smiles>

tert-Butyl 4-(2-acetyl-4-chlorophenyl)piperidine-1-carboxylate, 7a

mp 76-79 ${ }^{\circ} \mathrm{C} ;{ }^{-1} \mathrm{H}$ NMR $\left(\mathrm{CDCl}_{3}, 400 \mathrm{MHz}\right) \delta 7.53(\mathrm{~d}, J=2.3 \mathrm{~Hz}, 1 \mathrm{H}), 7.40(\mathrm{dd}, J=8.5,2.2 \mathrm{~Hz}, 1 \mathrm{H})$, 7.29 (d, $J=14.4 \mathrm{~Hz}, 1 \mathrm{H}), 4.22(\mathrm{br} \mathrm{s}, 2 \mathrm{H}), 3.22$ (tt, $J=12.0,3.4 \mathrm{~Hz}, 1 \mathrm{H}), 2.79($ app t, $J=11.9 \mathrm{~Hz}, 2 \mathrm{H}), 2.58$ (s, 3H), 1.79 (app d, $J=1.6 \mathrm{~Hz}, 2 \mathrm{H}), 1.64-1.52(\mathrm{~m}, 2 \mathrm{H}), 1.48(\mathrm{~s}, 9 \mathrm{H}) ;{ }^{13} \mathrm{C} \mathrm{NMR}\left(\mathrm{CDCl}_{3}, 100 \mathrm{MHz}\right) \delta$ 201.4, 154.7, 143.3, 139.8, 131.6, 131.2, 128.7, 128.1, 79.4, 44.2 (br), 37.7, 33.1, 30.3, 28.4; IR (thin film) 2975, 2927, 2844, $1689 \mathrm{~cm}^{-1}$; Anal. Calcd for $\mathrm{C}_{18} \mathrm{H}_{24} \mathrm{ClNO}_{3}$ : C, 63.99; H, 7.16; N, 4.15. Found: C, 63.73; $\mathrm{H}, 7.07 ; \mathrm{N}, 4.06$.<smiles>N#Cc1cc(Cl)ccc1Br</smiles>

\section{2-Bromo-5-chlorobenzonitrile, 8}

To a solution of 3-chlorobenzonitrile $(50 \mathrm{~g}, 360 \mathrm{mmol})$ in trifluoroacetic acid $(180 \mathrm{~mL})$ was added sulfuric acid $(24 \mathrm{~mL})$ and then 1,3-dibromo-5,5-dimethylhydantoin $(67 \mathrm{~g}, 234 \mathrm{mmol})$ in portions over $8 \mathrm{~min}$. The reaction temperature was allowed to reach $31^{\circ} \mathrm{C}$ and then cooling was applied to bring the temperature to $24^{\circ} \mathrm{C}$. After a $6 \mathrm{~h}$ age the heterogeneous reaction was cooled to $10^{\circ} \mathrm{C}$ and water $(250 \mathrm{~mL})$ was added. Following a 10 min ages, the reaction was filtered and the product cake was washed twice with water (250 and $100 \mathrm{~mL})$ to afford a white solid $(52.4 \mathrm{~g}, 63 \%)$. mp $137-140{ }^{\circ} \mathrm{C} ;{ }^{1} \mathrm{H} \mathrm{NMR}\left(\mathrm{CDCl}_{3}, 400 \mathrm{MHz}\right) \delta 7.64$ $7.62(\mathrm{~m}, 2 \mathrm{H}), 7.44(\mathrm{dd}, J=8.6,2.5 \mathrm{~Hz}, 1 \mathrm{H}) ;{ }^{13} \mathrm{C} \mathrm{NMR}\left(\mathrm{CDCl}_{3}, 100 \mathrm{MHz}\right) \delta 134.2,134.1,133.9,133.8$, 123.3, 117.2, 115.8; IR (thin film) 3086, 3052, $2228 \mathrm{~cm}^{-1}$; Anal. Calcd for $\mathrm{C}_{7} \mathrm{H}_{3} \mathrm{BrClN}$ : C, 38.84; $\mathrm{H}, 1.40$; N, 6.47. Found: C, 38.64; H, 1.18; N, 6.35 